TRANSACTIONS OF THE

AMERICAN MATHEMATICAL SOCIETY

Volume 360, Number 9, September 2008, Pages 4519-4540

S 0002-9947(08)04424-3

Article electronically published on April 4, 2008

\title{
FINITENESS OF HILBERT FUNCTIONS AND BOUNDS FOR CASTELNUOVO-MUMFORD REGULARITY OF INITIAL IDEALS
}

\author{
LÊ TUÂN HOA
}

Dedicated to Professor J. Herzog on the occasion of his 65th birthday

\begin{abstract}
Bounds for the Castelnuovo-Mumford regularity and Hilbert coefficients are given in terms of the arithmetic degree (if the ring is reduced) or in terms of the defining degrees. From this it follows that there exists only a finite number of Hilbert functions associated with reduced algebras over an algebraically closed field with a given arithmetic degree and dimension. A good bound is also given for the Castelnuovo-Mumford regularity of initial ideals which depends neither on term orders nor on the coordinates and holds for any field.
\end{abstract}

\section{INTRODUCTION}

In the famous book SGA6, Kleiman proved that given two positive integers $e$ and $d$, there exists only a finite number of Hilbert functions associated with reduced and equidimensional $K$-algebras $S$ over an algebraically closed field such that $\operatorname{deg} S \leq e$ and $\operatorname{dim} S=d$ (see $[\mathrm{K}$, Corollary 6.11]). An easier and more elegant proof of this result can be found in a recent paper by M. Rossi, N. V. Trung and G. Valla [RTV2]. Moreover, the paper [RTV2] gives a rather general approach for deriving the finiteness of Hilbert functions. It is shown that this problem (for a certain class of ideals) is equivalent to the boundedness of the Castelnuovo-Mumford regularity and the embedding dimension (see [RTV2, Theorem 2.3]). The first main purpose of this paper is to extend Kleiman's result to reduced $K$-algebras. A key point is to find a suitable invariant to replace the degree. Of course, a so-called extended degree is a choice, see [RVV] Corollary 4.4], but such an invariant is very large. It turns out that in our situation one can take the so-called arithmetic degree, a notion which maybe reflects better the complexity of ideals than the usual degree (see [BM, Section 3] and [V], Chapter 9]).

Theorem 0.1. Given two positive integers $a$ and $d$, assume that $K$ is an algebraically closed field. Then there exists only a finite number of Hilbert functions associated with reduced $K$-algebras $S$ such that $\operatorname{adeg} S \leq a$ and $\operatorname{dim} S=d$.

Received by the editors July 13, 2005.

2000 Mathematics Subject Classification. Primary 13D45, 13D40, 13 P10.

Key words and phrases. Castelnuovo-Mumford regularity, local cohomology, Hilbert function, Hilbert polynomial, initial ideal.

The author was supported in part by the National Basic Research Program (Vietnam). The final preparation of the article was done during his stay at the Centre de Recerca Matematica (Spain).

(C)2008 American Mathematical Society 4519

Reverts to public domain 28 years from publication 
Note that the above result does not hold for an arbitrary algebra (however see RVV, Corollary 4.4] and [RTV2, Theorem 3.1] for a possible generalization). As mentioned above, the main point in the proof of Theorem 0.1 is to bound the Castelnuovo-Mumford regularity. This is not hard to do (see Remark 1.6). However, a careful analysis allows us to establish the following explicit bound:

Theorem 0.2. Let $K$ be an arbitrary field and $I$ an arbitrary homogeneous ideal of $R=K\left[x_{1}, \ldots, x_{n}\right]$. Assume that $S=R / I$ is a reduced ring of dimension $d \geq 2$ and degree $e$. Then

$$
\operatorname{reg} I \leq\left(\frac{e(e-1)}{2}+\operatorname{adeg} I\right)^{2^{d-2}} .
$$

Applied to the case of reduced and equidimensional algebras, the bound of Theorem 0.2 is better than the one given in [RTV2, Theorem 3.1 and Lemma 3.3]. In view of the Eisenbud-Goto conjecture, the above bound is still too large, but it is a first explicit bound stated in terms of the arithmetic degree. In order to prove it, as in Lecture 14 of $\overline{\mathrm{M}}$ (see also $\overline{\mathrm{K}}, \overline{\mathrm{BM}}$ ) and $\mathrm{RTV2}$ ), we proceed by induction on the dimension. However, there is a different point: we simultaneously bound this invariant and the length of the graded components of certain local cohomology modules (see Theorem 1.5 and also Theorem 2.5).

The above technique can also be used to estimate the Castelnuovo-Mumford regularity of arbitrary homogeneous ideals in terms of the maximal degree $\Delta$ of minimal generators of $I \subset R=K\left[x_{1}, \ldots, x_{n}\right]$. If $K$ is any field of zero characteristic, from Giusti's paper [Gi] it follows that $\operatorname{reg}(I) \leq(2 \Delta)^{2^{n-2}}$. Bayer and Mumford suggested that this bound holds in any characteristic (see the comment after Theorem 3.7 in $[\mathrm{BM}]$ ). Not long ago, G. Caviglia and E. Sbarra proved that this is indeed the case:

$$
\operatorname{reg} I \leq\left(\Delta^{c}+\Delta c-c+1\right)^{2^{d-1}}
$$

where $c=n-d$ (see [CS, Corollary 2.6]). In this paper we will give a completely different proof for a slight improvement of this result (see Theorem 2.1).

The next problem we are interested in is to give good bounds for the CastelnuovoMumford regularity of initial ideals, in $(I)$, with respect to any term order and in any coordinates. Inspired by a result of Chardin and Moreno-Sosias, it was shown in $[\mathrm{HH}$ ] that if $R / I$ is a Cohen-Macaulay ring of multiplicity $e \geq 2$ and $d \geq 2$, then $\operatorname{reg}(\operatorname{in}(I)) \leq e^{2^{d-1}} / 2^{2^{d-2}}$. Long before that M. Giusti Gi showed that in characteristic zero we have $\operatorname{reg}(\operatorname{in}(I)) \leq(2 \Delta)^{2^{n-1}}$, provided the coordinates are chosen generically and the term order is the lexicographic order. Combining these facts with the above-mentioned result of [CS] and [MM, II.2.2], one may ask whether such a kind of bounds still holds for any $\operatorname{reg}(\operatorname{in}(I))$. Our second main result confirms it:

Theorem 0.3. Let $K$ be an arbitrary field and $I$ an arbitrary homogeneous ideal of $R$. With respect to any term order and any coordinates we have

$$
\operatorname{reg}(\operatorname{in} I) \leq\left(\frac{3}{2} \Delta^{c}+\Delta\right)^{d 2^{d-1}} .
$$

Moreover, if $R / I$ is a reduced algebra, then we also have

$$
\operatorname{reg}(\operatorname{in} I) \leq(\operatorname{adeg} I)^{(n-1) 2^{d-1}} .
$$


An immediate consequence of this theorem says that the maximal degree of a reduced Gröbner base, with respect to any term order and any coordinates, is bounded by $\left(\frac{3}{2} \Delta^{c}+\Delta\right)^{d 2^{d-1}}$. In view of a remarkable example due to Mayr and Meyer, this bound is nearly the best possible (see, e.g., Example 3.9 and Proposition 3.11 in $[\mathrm{BM}]$ ).

In order to prove this theorem we develop further the method in [HH]. Instead of initial ideals we consider a much larger class: the class of all ideals $J$ having the same Hilbert function as $I$. So, although the title of the paper is about initial ideals, we are in fact not dealing much with them. However, by doing so one can use Gotzmann's regularity theorem to bound $\operatorname{reg} J$ in terms of some data of $I$. Then, by virtue of Theorem 0.2 and Theorem 2.1, we will see that the only thing left is to estimate the Hilbert coefficients $e_{i}$ in terms of $\Delta$ or adeg $(I)$ (see Lemmas 6.1 and 6.3). This problem is also of independent interest. The main steps to doing it may be explained as follows. First, using a recent result by Herzog, Popescu and Vladoiu [HPV] one can bound cohomological Hilbert functions (i.e., the length of graded components of local cohomology modules) in terms of the CastelnuovoMumford regularity. From that we get bounds for the Hilbert coefficients by the Castelnuovo-Mumford regularity (see Theorems 4.1 and 4.6). The existence of such a bound was predicted by [RTV2, Theorem 2.3], and this approach is somewhat new, because usually one tries to estimate the latter invariant by the former ones (see, e.g., $\mathrm{K}$ and $\mathrm{BrS}$, Section 17.2]). However, it is a surprising fact that the relationships between these invariants in Theorems 4.1 and 4.6 are rather simple. Let us give here a simple version of these results.

Theorem 0.4. Let $e_{0}=e, \ldots, e_{d-1}$ be the Hilbert coefficents of $R / I$ and $b=$ $\max \{\Delta, \operatorname{adeg} I\}$. Then

(i) $\left|e_{1}\right| \leq b^{c} \operatorname{reg} I$.

(ii) For $i \geq 2,\left|e_{i}\right| \leq \frac{3}{2} b^{c}(\operatorname{reg} I)^{i}$.

Combining Theorems 4.1 and 4.6 with results on the Castelnuovo-Mumford regularity found earlier we get bounds for $\left|e_{i}\right|$ in terms of $\Delta$ or adeg $(I)$ (see Propositions 4.3 and 4.7). These bounds are huge: they are double exponential functions of $i$. But they are good enough to prove Theorem 0.3 Furthermore, Theorems 4.1 and 4.6 sometimes give really good bounds for $\left|e_{i}\right|$ if we already know a good estimation for the Castelnuovo-Mumford regularity (see Corollaries 4.4 and 4.8).

We now give a brief content of the paper. We prove Theorem 0.2 in Section 1 , and reprove in Section 2 the Caviglia-Sbarra bound on the Castelnuovo-Mumford regularity of an arbitrary homogeneous ideal in terms of the degrees of its defining equations (Theorem 2.1). Section 3 is devoted to bounding Hilbert cohomological functions in terms of the Castelnuovo-Mumford regularity (see Theorem 3.4). Bounds on Hilbert coefficients are given in Section 4. Putting results of the sections 1 and 4 together we are able to prove Theorem 0.1 without using RTV2]. This is done in Section 5 . Theorem 0.3 is proved in the last Section 6 . We refer the readers to Eisenbud's book [E] for unexplained terminology.

\section{Bounds in terms of the ARITHMetic DEgReE}

Throughout this paper, if not otherwise stated, $K$ is an arbitrary field, $R=$ $K\left[x_{1}, \ldots, x_{n}\right]$ is a polynomial ring and $I \subset R$ is a homogeneous ideal of dimension $d$. However, all invariants considered in this paper are not changed under passing 
from $K$ to $K(u)$, where $u$ is a new indeterminate. Hence, in proofs we may always assume that $K$ is an infinite field. This assumption guarantees the choice of generic elements.

Let $c=n-d$. Note that $c$ is the true codimension of $I$ if $I$ does not contain a linear form. Let $\mathfrak{m}=\left(x_{1}, \ldots, x_{n}\right)$ denote the maximal homogeneous ideal of $R$ and set $S=R / I$. Let us recall some notions.

For an Artinian $\mathbb{Z}$-graded module $N$, let

$$
\operatorname{end}(N)=\max \left\{t ; N_{t} \neq 0\right\}
$$

(with the convention $\max \emptyset=-\infty$ ). Further, let

$$
a_{i}(R / I)=\operatorname{end}\left(H_{\mathfrak{m}}^{i}(R / I)\right),
$$

where $H_{\mathfrak{m}}^{i}(R / I)$ is the local cohomology module with support in $\mathfrak{m}$ and $0 \leq i \leq d$. The Castelnuovo-Mumford regularity is the number

$$
\operatorname{reg}(R / I)=\max \left\{a_{i}(R / I)+i ; 0 \leq i \leq d\right\} .
$$

Note that $\operatorname{reg}(I)=\operatorname{reg}(R / I)+1$. Sometimes we also use the notation

$$
\operatorname{reg}_{k}(R / I)=\max \left\{a_{i}(R / I)+i ; k \leq i \leq d\right\},
$$

where $k$ is a nonnegative integer.

Following Brodmann and Sharp [BrS], the function

$$
h_{S}^{i}(t):=\ell\left(H_{\mathfrak{m}}^{i}(S)_{t}\right)
$$

is called the $i$-th Hilbert homological function of $S$, where $\ell($.) denotes the dimension of a vector space over $K$. Let $H_{S}(t)$ and $P_{S}(t)$ denote the Hilbert function and the Hilbert polynomial of $S$, respectively. We will often use the Grothendieck-Serre formula

$$
P_{S}(t)-H_{S}(t)=\sum_{i=0}^{d}(-1)^{i+1} h_{S}^{i}(t) .
$$

The leading coefficient of $P_{S}(t)$, multiplied by $(d-1)$ !, is called the degree of $S$ and denoted by $\operatorname{deg} S$. We also denote $\operatorname{deg} S$ by $e(S)$, or just by $e$. The arithmetic degree is defined as follows:

$$
\operatorname{adeg} S=\operatorname{adeg} I=\sum_{\mathfrak{p} \in \operatorname{Ass}(R / I)} \ell\left(H_{\mathfrak{m}_{\mathfrak{p}}}^{0}\left(R_{\mathfrak{p}} / I_{\mathfrak{p}}\right)\right) \operatorname{deg}(R / \mathfrak{p})
$$

(see [BM, Definition 3.4] and [V, Definition 9.13]). The number $\ell\left(H_{\mathfrak{m}_{\mathfrak{p}}}^{0}\left(R_{\mathfrak{p}} / I_{\mathfrak{p}}\right)\right)$ is the multiplicity of the component $\mathfrak{p}$ with respect to $I$. In this definition $\mathfrak{p}$ runs over all associated primes of $S$, while the usual degree $\operatorname{deg} S$ can be computed by a similar formula, but the sum is only taken over primes of the highest dimension. Thus

$$
\operatorname{adeg} S \geq \operatorname{deg} S
$$

and the equality holds if and only if $S$ is a pure-dimensional ring.

In this section we prove Theorem 0.2 . We need some auxiliary results.

Lemma 1.1. Let $S$ be a one-dimensional Cohen-Macaulay ring. Then

$$
h_{S}^{1}(0)+\cdots+h_{S}^{1}(\operatorname{reg} S-1) \leq e(e-1) / 2 .
$$


Proof. Since $P_{S}(t)=e$, from the Grothendieck-Serre formula (2) we have

$$
h_{S}^{1}(t)=e-H_{S}(t) .
$$

Let $r=\operatorname{reg} S$. Since $S$ is a Cohen-Macaulay ring, its Hilbert-Poincaré series can be written in the form

$$
H P_{S}(z):=\sum_{i \geq 0} H_{S}(i) z^{i}=\frac{1+h_{1} z+\cdots+h_{r} z^{r}}{1-z}
$$

where $h_{1}, \ldots, h_{r}$ are positive integers (see, e.g., [V, p. 240]). From this it follows that

$$
H_{S}(t)=1+h_{1}+\cdots+h_{t} \geq t+1
$$

for all $t \leq r$. Moreover, under the Cohen-Macaulay assumption, $r \leq e-1$. Hence

$$
h_{S}^{1}(0)+\cdots+h_{S}^{1}(\operatorname{reg} S-1) \leq r e-(1+\cdots+r)=r(2 e-r-1) / 2 \leq e(e-1) / 2 .
$$

Lemma 1.2. Assume that $S=R / I$ is a reduced ring of dimension at least two. Then

$$
h_{S}^{1}(-1) \leq \operatorname{adeg} I-e .
$$

Proof. Since $S$ is reduced, one may write $I=J \cap Q$, where $J$ is the intersection of all associated primes of $R / I$ of dimension at least 2 , and $Q$ is the intersection of all associated primes of $R / I$ of dimension 1. By [HSV, Lemma 1] we have $h_{R / J}^{1}(-1)=$ 0 . Thus if $Q=R$, then $h_{S}^{1}(-1)=0$. Assume that $Q \neq R$. Since $J \neq R$ and $R / I$ has no embedded primes, $J+Q$ is an $\mathfrak{m}$-primary ideal, i.e. $\operatorname{dim} R /(J+Q)=0$. The exact sequence

$$
0 \rightarrow S \rightarrow R / J \oplus R / Q \rightarrow R /(J+Q) \rightarrow 0
$$

implies

$$
h_{S}^{1}(-1)=h_{R / J}^{1}(-1)+h_{R / Q}^{1}(-1)=h_{R / Q}^{1}(-1) .
$$

Note that $\operatorname{deg} R / Q=\operatorname{adeg} I-\operatorname{adeg} J \leq \operatorname{adeg} I-e$. Since $R / Q$ is a one-dimensional ring, by the Grothendieck-Serre formula, we have

$$
h_{R / Q}^{1}(-1)=\operatorname{deg} R / Q \leq \operatorname{adeg} I-e .
$$

The proof of Theorem 0.2 proceeds by induction. The next two lemmas allow us to do induction. The first one is concerned with the behavior of the arithmetic degree by hyperplane section. It is more subtle than the usual degree; see [MVY]. However we have

Lemma 1.3. Let $K$ be an infinite field, and $S=R / I$ an arbitrary ring of dimension at least two and positive depth. Assume that $x_{n}$ is chosen generically. Let $T=$ $R /\left(\left(I, x_{n}\right): \mathfrak{m}^{\infty}\right)$. Then:

(i) $\operatorname{reg} T \leq \operatorname{reg} S$,

(ii) $\operatorname{adeg} T \leq \operatorname{adeg} S$. 
Proof. (i) Since $x_{n}$ is generic, it is a regular element of $S$. We have

$$
\operatorname{reg} T=\operatorname{reg}_{1} S / x_{n} S \leq \operatorname{reg} S / x_{n} S=\operatorname{reg} S .
$$

(ii) For an $R$-module $M$ and $r \geq-1$, let

$$
\operatorname{adeg}_{r}(M)=\sum_{\mathfrak{p} \in \operatorname{Ass}(M), \operatorname{dim} R / \mathfrak{p}=r+1} \ell\left(H_{\mathfrak{m}_{\mathfrak{p}}}^{0}\left(M_{\mathfrak{p}}\right)\right) \operatorname{deg}(R / \mathfrak{p})
$$

(see [BM, Definition 3.4]). Since $x_{n}$ is generic, by the prime avoidance lemma, we may assume

$$
x_{n} \notin \bigcup\left\{\mathfrak{p} ; \mathfrak{m} \neq \mathfrak{p} \in \operatorname{Ass}(S) \bigcup_{j \geq 1} \operatorname{Ass}\left(\operatorname{Ext}_{R}^{n-j}(S, R)\right)\right\} .
$$

By [MVY, Corollary 2.5] it follows that

$$
\operatorname{adeg}_{r-1}(T)=\operatorname{adeg}_{r}(S) \text { for all } r \geq 1 .
$$

Since $S$ and $T$ have no zero-dimensional component, we get

$$
\begin{aligned}
\operatorname{adeg} T & =\operatorname{adeg}_{0}(T)+\cdots+\operatorname{adeg}_{d-1}(T) \\
& =\operatorname{adeg}_{1}(S)+\cdots+\operatorname{adeg}_{d}(S) \leq \operatorname{adeg} S .
\end{aligned}
$$

The first three statements of the next lemma are contained in the proof of Mumford's theorem on page 101 of the book [M] (cf. also [K, Proposition 1.4], [RTV1, Theorem 1.4] and [RTV2, Theorem 1.3]). In order to make the paper more selfcontained, we give here a sketch of the proof. The proof of (iii) here is also simpler.

Lemma 1.4. Let $K$ be an infinite field and $S=R / I$ a reduced ring of dimension at least two. Assume that $x_{n}$ is chosen generically. Let $T=R /\left(\left(I, x_{n}\right): \mathfrak{m}^{\infty}\right)$ and $r=\operatorname{reg} T$. Then $T$ is also a reduced ring and we have

(i) $\operatorname{reg}_{2}(S) \leq r$ (see the definition in (10)),

(ii) $h_{S}^{1}(t) \geq h_{S}^{1}(t+1)$ for all $t \geq r-1$,

(iii) $\operatorname{reg} S \leq r+h_{S}^{1}(r-1)$,

(iv) $h_{S}^{1}(t) \leq h_{T}^{1}(0)+\cdots+h_{T}^{1}(t)+\operatorname{adeg} I-e$, for all $t \geq 0$.

Proof. Note that $T$ can be considered as the homogeneous coordinate ring of a generic hyperplane section of the scheme $\operatorname{Proj}(S)$. Since $K$ is an infinite ring and $x_{n}$ is generic, by Bertini's theorem [FOV], Corollary 3.4.14] it follows that $T$ is reduced.

The long exact sequence

$$
\begin{aligned}
0 \rightarrow H_{\mathfrak{m}}^{0}\left(S / x_{n} S\right)_{t} & \rightarrow H_{\mathfrak{m}}^{1}(S)_{t-1} \rightarrow H_{\mathfrak{m}}^{1}(S)_{t} \stackrel{\varphi_{t}}{\longrightarrow} H_{\mathfrak{m}}^{1}\left(S / x_{n} S\right)_{t}=H_{\mathfrak{m}}^{1}(T)_{t} \\
& \rightarrow H_{\mathfrak{m}}^{2}(S)_{t-1} \rightarrow H_{\mathfrak{m}}^{2}(S)_{t} \rightarrow \cdots
\end{aligned}
$$

implies (i) and the short exact sequence

$$
0 \rightarrow H_{\mathfrak{m}}^{0}\left(S / x_{n} S\right)_{t} \rightarrow H_{\mathfrak{m}}^{1}(S)_{t-1} \rightarrow H_{\mathfrak{m}}^{1}(S)_{t} \rightarrow 0
$$

for all $t \geq r$. This yields (ii). If $h_{S}^{1}\left(t_{0}-1\right)=h_{S}^{1}\left(t_{0}\right)$ for some $t_{0} \geq r+1$, we would have $h_{S / x_{n} S}^{0}\left(t_{0}\right)=0$. Since $\operatorname{reg}_{1}\left(S / x_{n} S\right)=\operatorname{reg} T=r$, it then implies that $\operatorname{reg}\left(S / x_{n} S\right) \leq t_{0}$. Hence $h_{S}^{1}\left(t_{0}\right)=h_{S}^{1}\left(t_{0}+1\right)=\cdots=0$. Therefore $h_{S}^{1}(t)$ is strictly decreasing to zero when $t \geq r$, which implies (iii).

It remains to show (iv). From the exact sequence (3) we have

$$
h_{S}^{1}(u)-h_{S}^{1}(u-1)=\ell\left(\operatorname{Im}\left(\varphi_{u}\right)\right)-h_{S / x_{n} S}^{0}(u) \leq h_{T}^{1}(u)
$$


for all $u \in \mathbb{Z}$. Adding these inequalities and using Lemma 1.2 we get

$$
\begin{aligned}
h_{S}^{1}(t) & \leq h_{T}^{1}(0)+\cdots+h_{T}^{1}(t)+h_{S}^{1}(-1) \\
& \leq h_{T}^{1}(0)+\cdots+h_{T}^{1}(t)+\operatorname{adeg} I-e
\end{aligned}
$$

for all $t \geq 0$.

Theorem 0.2 is a part of the following result. If $a \in \mathbb{R}$, we denote by $[a]$ the largest integer not exceeding $a$.

Theorem 1.5. Assume that $S=R / I$ is a reduced ring of dimension at least two. Let

$$
m=\frac{e(e-1)}{2}+\operatorname{adeg} I .
$$

Then

(i) $\operatorname{reg} S \leq m^{2^{d-2}}-1$,

(ii) for all $t \geq 0$, we have $h_{S}^{1}(t) \leq m^{2^{d-2}}-e \cdot m^{\left[2^{d-3}\right]}$.

Proof. We may assume that $x_{n}$ is generic and choose $T$ as in the previous lemma. Hence $T$ is a reduced ring. Set $r=\operatorname{reg} T$.

Let $d=2$. In order to show (ii), by Lemma 1.4(ii), we may assume that $t \leq r-1$. Note that $T$ is a Cohen-Macaulay ring and $e(T)=e$. Then Lemma 1.4(iv) and Lemma 1.1 yield:

$$
\begin{aligned}
h_{S}^{1}(t) & \leq h_{T}^{1}(0)+\cdots+h_{T}^{1}(t)+\operatorname{adeg} I-e \\
& \leq h_{T}^{1}(0)+\cdots+h_{T}^{1}(r-1)+\operatorname{adeg} I-e \\
& \leq \frac{e(e-1)}{2}+\operatorname{adeg} I-e=m-e .
\end{aligned}
$$

Using this inequality and the fact that $r \leq e-1$ (since $T$ is a Cohen-Macaulay ring), by Lemma 1.4(iii) we get

$$
\operatorname{reg} S \leq e-1+m-e=m-1 .
$$

Thus the case $d=2$ is proven.

Let $d \geq 3$. Since $\operatorname{dim} T=d-1, e(T)=e$, and $\operatorname{adeg} T \leq \operatorname{adeg} S$ (by Lemma $1.3($ ii) $)$, the induction hypothesis gives

$$
r \leq m^{2^{d-3}}-1
$$

and for all $t \geq 0$,

$$
h_{T}^{1}(t) \leq m^{2^{d-3}}-e \cdot m^{\left[2^{d-4}\right]} \leq m^{2^{d-3}}-e .
$$

In order to prove (ii), again by Lemma 1.4(ii), we may assume that $t \leq r-1$. Then, by Lemma 1.4(iv), for all $t \geq 0$ we have

$$
\begin{aligned}
h_{S}^{1}(t) & \leq h_{T}^{1}(0)+\cdots+h_{T}^{1}(t)+\operatorname{adeg} I-e \\
& \leq r\left(m^{2^{d-3}}-e\right)+\operatorname{adeg} I-e \quad(\operatorname{by} \quad(50)) \\
& \leq\left(m^{2^{d-3}}-1\right)\left(m^{2^{d-3}}-e\right)+\operatorname{adeg} I-e \quad(\text { by (44) }) \\
& =m^{2^{d-2}}-e \cdot m^{2^{d-3}}-m^{2^{d-3}}+\operatorname{adeg} I \\
& \leq m^{2^{d-2}}-e \cdot m^{2^{d-3}} .
\end{aligned}
$$


To prove (i) we use (ii) and Lemma 1.4(iii) :

$$
\operatorname{reg} S \leq r+h_{S}^{1}(r-1) \leq m^{2^{d-3}}-1+m^{2^{d-2}}-e \cdot m^{2^{d-3}} \leq m^{2^{d-2}}-1 .
$$

Remark 1.6. In order to get a bound for reg $S$ in terms of adeg $S$ and $d$, by induction on $d$, it suffices to estimate $h_{S}^{1}(t)$ for $t \geq 0$. This can be easily done by using the following well-known inequality:

$$
h_{S}^{1}(t)+H_{S}(t) \leq \operatorname{adeg} S\left(\begin{array}{c}
t+d-1 \\
d-1
\end{array}\right)
$$

(recall that $S$ is reduced). This was pointed out by the referee. However a direct application of this inequality would only lead to a bound of the following type:

$$
\operatorname{reg} I \leq(\operatorname{adeg} I)^{d-1) !} .
$$

If $K$ is an algebraically closed field, then a result of Gruson, Lazarsfeld and Peskine GLP yields a better bound for the case $d=2$ as shown in the following statement.

Proposition 1.7. Let $K$ be an algebraically closed field. Assume that $R / I$ is a reduced ring of dimension two. Then $\operatorname{reg} I \leq \operatorname{adeg} I$.

Proof. Write $I=J \cap Q$ as in the proof of Lemma 1.2, Then we have an exact sequence:

$$
0 \rightarrow H_{\mathfrak{m}}^{0}(R / J+Q)_{t} \rightarrow H_{\mathfrak{m}}^{1}(R / I)_{t} \rightarrow H_{\mathfrak{m}}^{1}(R / J)_{t} \oplus H_{\mathfrak{m}}^{1}(R / Q)_{t} \rightarrow 0,
$$

and

$$
H_{\mathfrak{m}}^{2}(R / I)_{t} \cong H_{\mathfrak{m}}^{2}(R / J)_{t} \oplus H_{\mathfrak{m}}^{2}(R / Q)_{t} .
$$

By [GLP, Theorem 1.1] (see also the remark on p. 497 there), reg $R / J \leq e-1 \leq$ adeg $I-1$. So we may assume that $Q \neq R$. Since $R / Q$ is one-dimensional and reduced, it is a Cohen-Macaulay ring. Hence $\operatorname{reg} R / Q \leq \operatorname{adeg} R / Q-1<\operatorname{adeg} I$. To complete the proof it suffices to show that

$$
H_{\mathfrak{m}}^{0}(R / J+Q)_{t}=0 \text { for all } t \geq \operatorname{adeg} I-1 .
$$

Since reg $J \leq e, J$ is generated by elements of degree $\leq e$. Hence one may choose an element $x \in J$ of degree $e$ such that $x$ does not belong to any prime in $Q$; i.e., $x$ is a regular element on $R / Q$. Then we have

$$
\operatorname{reg} R /(Q, x)=\operatorname{reg} R / Q+e-1 \leq \operatorname{deg} R / Q-1+e-1=\operatorname{adeg} I-2 .
$$

Since $R /(Q, x)$ is a zero-dimensional ring, this means $(R /(Q, x))_{t}=0$ for all $t \geq$ adeg $I-1$. The inequality $\ell\left((R / J+Q)_{t}\right) \leq \ell\left((R /(Q, x))_{t}\right)$ gives us $H_{\mathfrak{m}}^{0}(R / J+Q)_{t}=$ $(R / J+Q)_{t}=0$ for all $t \geq \operatorname{adeg} I-1$, as required.

The above proposition says that in dimension two one can replace $m$ by adeg $S$ in Theorem[1.5(i). However this does not work for Theorem[1.5(ii) as shown by the following example.

Example 1.8. Given $e \geq 6$ and $S=K\left[x^{e}, x^{e-1} y, x y^{e-1}, y^{e}\right]$, then for $0 \leq t \leq e-2$ one can show that $h_{S}^{1}(t)=t e+1-(t+1)^{2}$, while adeg $S-e=0$. Taking $t_{0}=\left[\frac{e-2}{2}\right]$, one can see that $h_{S}^{1}\left(t_{0}\right)$ is approximately half of the bound in Theorem 1.5)(ii).

Theorem 0.2 does not hold if the ring $R / I$ is not reduced. 
Example 1.9 (see [V] Example 9.3.1]). Let $S=K[x, y, u, v] /\left((x, y)^{2}, x u^{t}+y v^{t}\right)$, $t \geq 1$. Then adeg $S=e=2$, while reg $S=t$ can be arbitrarily large.

\section{Bounds In TERMS OF DEGREES OF DEFINING EQUATIONS}

In this section we study arbitrary homogeneous ideals. We will always write the degrees of polynomials in a minimal homogeneous basis of $I$ in a decreasing sequence

$$
\Delta:=\delta_{1} \geq \delta_{2} \geq \cdots
$$

and assume $\Delta \geq 2$. As mentioned in the introduction, G. Caviglia and E. Sbarra already proved that

$$
\operatorname{reg} I \leq\left(\Delta^{c}+\Delta c-c+1\right)^{2^{d-1}}
$$

(see [CS, Corollary 2.6]). The purpose of this section is to prove the following theorem, which is a slight improvement of the above result.

Theorem 2.1. Let $K$ be an arbitrary field and I be an arbitrary homogeneous ideal of dimension $d \geq 1$. Then

$$
\operatorname{reg} I \leq\left(\delta_{1} \cdots \delta_{c}+\Delta-1\right)^{2^{d-1}} \leq\left(\Delta^{c}+\Delta-1\right)^{2^{d-1}} .
$$

The proof of [CS] uses properties of Borel-fixed ideals. The proof here is completely different and simpler than the one in [CS]. The main idea of the proof is similar to that of Theorem 0.2 . We need some technical lemmas. For short, set

$$
\sigma=\delta_{1}+\cdots+\delta_{c}-c \text { and } \pi=\delta_{1} \cdots \delta_{c} .
$$

If $S=R / I$, then we also write $\Delta=\Delta(S), \delta_{1}=\delta_{1}(S), \ldots$ to emphasize their dependence on $S$ (or $I$ ). The following result was pointed out to the author by the referee. Subsequently, it slightly improves our original Theorem 2.5 and Theorem 6.4 .

Lemma 2.2 ([Sj], Theorem 2]). If $\operatorname{dim} S \leq 1$, then $\operatorname{reg} S \leq \sigma+\Delta-1$.

The next result is a special case of [HH, Lemma 3].

Lemma 2.3. Assume that $d=1$. Then for all $t \geq 1, h_{S}^{0}(t) \leq \pi-1$.

Recall that an element $x \in \mathfrak{m}$ is called filter regular if $0: \mathfrak{m}^{\infty}$ is of finite length.

Lemma 2.4. Assume that $\operatorname{dim} S \geq 1$ and $x=x_{n}$ is a filter regular element on $S$. Let $T=S / x S$ and $r \geq \max \{\operatorname{reg} T, \Delta-1\}$. Then

(i) $\operatorname{reg}_{1}(S) \leq r$ (see the definition in (10)),

(ii) $h_{S}^{0}(t) \geq \bar{h}_{S}^{0}(t+1)$ for all $t \geq r$,

(iii) $\operatorname{reg} S \leq r+h_{S}^{0}(r)$,

(iv) $h_{S}^{0}(t) \leq h_{T}^{0}(1)+\cdots+h_{T}^{0}(t)$, for all $t \geq 1$.

Proof. (i)-(iii) were shown in the proof of [BM, Proposition 3.8]. It follows from the following exact sequence:

$$
0 \rightarrow(0: x)_{t-1} \rightarrow H_{\mathfrak{m}}^{0}(S)_{t-1} \rightarrow H_{\mathfrak{m}}^{0}(S)_{t} \stackrel{\varphi_{t}}{\longrightarrow} H_{\mathfrak{m}}^{0}(T)_{t} \rightarrow H_{\mathfrak{m}}^{1}(S)_{t-1} \rightarrow H_{\mathfrak{m}}^{1}(S)_{t} \rightarrow \cdots
$$

For (iii) we need also the assumption $r \geq \Delta-1$ in order to apply the regularity criterion of [BS, Theorem 1.10].

From the above exact sequence we have

$$
h_{S}^{0}(u)-h_{S}^{0}(u-1)=\ell\left(\operatorname{Im}\left(\varphi_{u}\right)\right)-\ell\left((0: x)_{u-1}\right) \leq h_{T}^{0}(u)
$$

for all $u \in \mathbb{Z}$. Since $h_{S}^{0}(0)=0$, adding these inequalities gives us (iv). 
Theorem 2.1 is a part of the following

Theorem 2.5. Let $d \geq 1$. Then

(i) $\operatorname{reg} S \leq(\pi+\Delta-1)^{2^{d-1}}-1$.

(ii) For all $t \geq 1$, we have $h_{S}^{0}(t) \leq(\pi+\Delta-1)^{2^{d-1}}-(\pi+\Delta-1)^{\left[2^{d-2}\right]}$.

Proof. Keep the notation of Lemma 2.4 and let $I^{\prime}$ denote the image of $I$ in $K\left[x_{1}, \ldots, x_{n}\right] /\left(x_{n}\right) \cong K\left[x_{1}, \ldots, x_{n-1}\right]=: R^{\prime}$. Then $T \cong R^{\prime} / I^{\prime}$ and it is clear that $\sigma\left(I^{\prime}\right) \leq \sigma$ and $\pi\left(I^{\prime}\right) \leq \pi$.

First let $d=1$. With the above remark, Lemma 2.3 yields $h_{S}^{0}(t) \leq \pi-1$ for all $t \geq 1$. Thus (ii) holds. By Lemma 2.2.

$$
\operatorname{reg} S \leq \delta_{1}+\delta_{2}+\cdots+\delta_{c}-c+\Delta-1 .
$$

By induction on $c$ we get

$$
\operatorname{reg} S \leq\left(\delta_{1} \cdots \delta_{c-1}-1\right)+\left(\delta_{c}-1\right)+\Delta-1 \leq \delta_{1} \cdots \delta_{c}-1+\Delta-1=\pi+\Delta-2 .
$$

Thus the case $d=1$ is proven.

Now let $d \geq 2$. With the remark at the beginning of the proof and by the induction hypothesis we may assume that

$$
\operatorname{reg} T \leq(\pi+\Delta-1)^{2^{d-2}}-1
$$

and

$$
h_{T}^{0}(t) \leq(\pi+\Delta-1)^{2^{d-2}}-(\pi+\Delta-1)^{\left[2^{d-3}\right]} \leq(\pi+\Delta-1)^{2^{d-2}}-1 .
$$

Let $r=(\pi+\Delta-1)^{2^{d-2}}$. Obviously $r \geq \Delta$. In order to prove (i), by Lemma 2.4(ii), we may assume that $t \leq r$. Then, by Lemma 2.4 (iv) and the induction hypothesis, we have

$$
\begin{aligned}
h_{S}^{0}(t) & \leq r\left((\pi+\Delta-1)^{2^{d-2}}-1\right) \leq(\pi+\Delta-1)^{2^{d-2}}\left((\pi+\Delta-1)^{2^{d-2}}-1\right) \\
& =(\pi+\Delta-1)^{2^{d-1}}-(\pi+\Delta-1)^{2^{d-2}} .
\end{aligned}
$$

Thus (ii) is proven. Using this and Lemma 2.4(iii) we immediately get (i).

Remark 2.6. a) The bound in Theorem 2.1 is nearly the best possible. It was shown that there is an ideal $I$, due to Mayr and Meyer, generated by $10 n-6$ forms of degree at most 4 in $10 n+1$ variables such that $\operatorname{reg}(I)>4^{2^{n-1}}+1$ (see, e.g., $\mathrm{BM}$, Example 3.9 and Proposition 3.11]).

b) In this paper we are not interested in giving the best possible bounds for $\operatorname{reg} S$, which are then more complicated to formulate. On the other hand, for rings of small dimension, there are also some bounds which are much better than the ones in Theorem 2.1. See, e.g., a recent paper $\overline{\mathrm{CF}}$ for $d \leq 2$. However an application of such results to our proof does not significantly improve the bound in Theorem 2.5 for a larger $d$.

\section{Hilbert cohomological functions}

In this section we give a bound on $h_{S}^{i}(t)$. First, we do this for Borel-fixed ideals. We need some notation and results from [HPV]. Let $I \neq 0$ be a monomial ideal. Denote by $G(I)$ the unique set of monomial generators of $I$. For a monomial $u$, let $m(u)$ be the maximal index of a variable appearing in $u$. Set

$$
m(I)=\max \{m(u) ; u \in G(I)\} .
$$


We recursively define an ascending chain of monomial ideals

$$
I=I_{0} \subset I_{1} \subset \cdots \subset I_{l+1}=R
$$

as follows: let $I_{0}=I$. Suppose $I_{j}$ is already defined. If $I_{j}=R$, then the chain ends. Otherwise, let $n_{j}=m\left(I_{j}\right)$ and set

$$
I_{j+1}=I_{j}: x_{n_{j}}^{\infty}:=\bigcup_{k=1}^{\infty} I_{j}: x_{n_{j}}^{k} .
$$

A stable ideal under the action of upper triangular matrices is called Borel-fixed. It is always a monomial ideal. If $I$ is a Borel-fixed ideal, then $\left(x_{1}, \ldots, x_{c}\right)$ is the unique minimal associated prime of $R / I$ (see [E, Corollary 15.25]). Hence in this case, $n \geq n_{0}>n_{1}>\cdots>n_{l}=c$. For $j=0, \ldots, l$, let $J_{j} \subset K\left[x_{1}, \ldots, x_{n_{j}}\right]$ be the monomial ideal with $G\left(I_{j}\right)=G\left(J_{j}\right)$. Denote by

$$
J_{j}^{s a t}=J_{j}:\left(x_{1}, \ldots, x_{n_{j}}\right)^{\infty}
$$

the saturation of $J_{j}$. Then by [HPV, Corollary 2.6] and local duality we have

Lemma 3.1. Let $I \neq 0$ be a Borel-fixed ideal. Then $H_{\mathfrak{m}}^{j}(S)=0$ if $j \notin\{n-$ $\left.n_{0}, \ldots, n-n_{l}\right\}$, and we have an isomorphism of $\mathbb{Z}$-graded $R$-modules:

$$
H_{\mathfrak{m}}^{j}(S) \cong\left(J_{i}^{s a t} / J_{i}\right)\left[x_{n_{i}+1}^{-1}, \ldots, x_{n}^{-1}\right],
$$

if $j=n-n_{i}$ for some $i=0, \ldots, l$.

In the sequel, for a Borel-fixed ideal $I$ let us denote

$$
B:=B(I)=\ell\left(R /\left(I, x_{c+1}, \ldots, x_{n}\right)\right) .
$$

For short, set $e=\operatorname{deg}(I)$. Note that $B \geq e$.

Lemma 3.2. Let $I \neq 0$ be a Borel-fixed ideal. Then

(i) $\ell\left(J_{l}^{\text {sat }} / J_{l}\right)=e$.

(ii) For $i<l$ and all $t \geq 0$ we have

$$
\ell\left(\left[J_{i}^{s a t} / J_{i}\right]_{t}\right) \leq(B-1)\left(\begin{array}{c}
t+n_{i}-c-2 \\
n_{i}-c-1
\end{array}\right) .
$$

Proof. Let $M=J_{i}^{\text {sat }} / J_{i}$ and $R^{\prime}=K\left[x_{1}, \ldots, x_{c}\right]$. Since $\left(x_{1}, \ldots, x_{c}\right)$ is the unique minimal associated prime of $R / I$, by the construction we have $I \subseteq I_{i} \subseteq\left(x_{1}, \ldots, x_{c}\right)$.

Let $i=l$. We have $J_{l}^{\text {sat }}=R^{\prime}$ and $J_{l}=I:\left(x_{c+1}, \ldots, x_{n}\right)^{\infty}$. Hence

$$
\ell(M)=\ell\left(R^{\prime} / I:\left(x_{c+1}, \ldots, x_{n}\right)^{\infty}\right)=\ell\left((R / I)_{\left(x_{c+1}, \ldots, x_{n}\right)}\right)=e .
$$

Let $i<l$. Set $R^{\prime \prime}=K\left[x_{1}, \ldots, x_{n_{i}}\right]$. By the definition $J_{i}=G\left(I_{i}\right) R^{\prime \prime}$. Hence $x_{c+1}, \ldots, x_{n_{i}}$ is an s.o.p. of $R^{\prime \prime} / J_{i}$, and $I \cap R^{\prime} \subseteq J_{i} \cap R^{\prime}$. This implies

$$
\ell\left(\frac{R^{\prime \prime}}{\left(J_{i}, x_{c+1}, \ldots, x_{n_{i}}\right)}\right)=\ell\left(\frac{R^{\prime}}{J_{i} \cap R^{\prime}}\right) \leq \ell\left(\frac{R^{\prime}}{I \cap R^{\prime}}\right)=\ell\left(\frac{R}{\left(I, x_{c+1}, \ldots, x_{n}\right)}\right)=B .
$$

On the other side, the inclusion $J_{i}^{\text {sat }} \subseteq\left(x_{1}, \ldots, x_{c}\right)$ yields

$$
\ell\left(M_{t}\right) \leq \ell\left(\frac{\left(x_{1}, \ldots, x_{c}\right) R^{\prime \prime}}{J_{i}}\right)=\ell\left(\frac{R^{\prime \prime}}{J_{i}}\right)-\ell\left(\frac{R^{\prime \prime}}{\left(x_{1}, \ldots, x_{c}\right) R^{\prime \prime}}\right)=\ell\left(\frac{R^{\prime \prime}}{J_{i}}\right)-\left(\begin{array}{c}
t+n_{i}-c-1 \\
n_{i}-c-1
\end{array}\right) .
$$


By [RVV, Proposition 2.4] and (7) we have

$$
\begin{aligned}
\ell\left(\frac{R^{\prime \prime}}{J_{i}}\right) & \leq\left(\ell\left(\frac{R^{\prime \prime}}{\left(J_{i}, x_{c+1}, \ldots, x_{n_{i}}\right)}\right)-1\right)\left(\begin{array}{c}
t+n_{i}-c-2 \\
n_{i}-c-1
\end{array}\right)+\left(\begin{array}{c}
t+n_{i}-c-1 \\
n_{i}-c-1
\end{array}\right) \\
& \leq(B-1)\left(\begin{array}{c}
t+n_{i}-c-2 \\
n_{i}-c-1
\end{array}\right)+\left(\begin{array}{c}
t+n_{i}-c-1 \\
n_{i}-c-1
\end{array}\right) .
\end{aligned}
$$

Hence $\ell\left(M_{t}\right) \leq(B-1)\left(\begin{array}{c}t+n_{i}-c-2 \\ n_{i}-c-1\end{array}\right)$.

Lemma 3.3. Let $I \neq 0$ be a Borel-fixed ideal and $S=R / I$. Then

(i) $h_{S}^{0}(t) \leq(B-1)\left(\begin{array}{c}t+d-2 \\ d-1\end{array}\right)$ for all $t \geq 0$.

(ii) For $1 \leq j \leq d-1$ and $t \leq \operatorname{reg} S$ :

$$
h_{S}^{j}(t) \leq(B-1)\left(\begin{array}{c}
\operatorname{reg} S+d-j-2 \\
d-j-1
\end{array}\right)\left(\begin{array}{c}
\operatorname{reg} S-t \\
j
\end{array}\right) .
$$

(iii) For $t<\operatorname{reg} S$ :

$$
h_{S}^{d}(t) \leq e\left(\begin{array}{c}
\operatorname{reg} S-t-1 \\
d-1
\end{array}\right) \leq B\left(\begin{array}{c}
\operatorname{reg} S-t-1 \\
d-1
\end{array}\right) .
$$

Proof. By virtue of Lemma 3.1 (i) is a special case of Lemma 3.2 (when $i=0$ ). Let $j \geq 1$. By Lemma 3.1 we may assume that $j=n-n_{i}$ for some $i>0$. Let $M=J_{i}^{\text {sat }} / J_{i}$. Lemma 3.1 implies

$$
\begin{aligned}
h_{S}^{j}(t) & =\sum_{u=1}^{\operatorname{end}(M)} \ell\left(M_{u}\right)\left(\begin{array}{c}
u-j-t+j-1 \\
j-1
\end{array}\right) \\
& \leq\left[\max _{1 \leq u \leq \operatorname{end}(M)} \ell\left(M_{u}\right)\right] \sum_{v=1}^{\operatorname{end}(M)-j-t}\left(\begin{array}{c}
v+j-1 \\
j
\end{array}\right) \\
& =\left[\max _{1 \leq u \leq \operatorname{end}(M)} \ell\left(M_{u}\right)\right]\left(\begin{array}{c}
\operatorname{end}(M)-t \\
j
\end{array}\right) .
\end{aligned}
$$

(In the above calculation we set $\left(\begin{array}{l}a \\ b\end{array}\right)=0$ if $b \geq 0$ and $a<b$.) Moreover, again by Lemma 3.1, end $(M)=a_{j}(S)+j \leq \operatorname{reg} S$ (see also [HPV, Corollary 2.7]). Since $n_{i}-c=n-j-c=d-j$, Lemma 3.2 (ii) yields

$\max _{1 \leq u \leq \operatorname{end}(M)} \ell\left(M_{u}\right) \leq(B-1) \max _{1 \leq u \leq \operatorname{reg} S}\left(\begin{array}{c}u+d-j-2 \\ d-j-1\end{array}\right)=(B-1)\left(\begin{array}{c}\operatorname{reg} S+d-j-2 \\ d-j-1\end{array}\right)$.

From this and (9) we get (ii).

Let $j=d$. Then $n_{i}=c$. From (8) we have

$$
\begin{aligned}
h_{S}^{d}(t) & \leq\left[\max _{1 \leq u \leq \operatorname{end}(M)}\left(\begin{array}{c}
u-t-1 \\
d-1
\end{array}\right)\right] \cdot \sum_{u=1}^{\operatorname{end}(M)} \ell\left(M_{u}\right) \\
& \leq\left(\begin{array}{c}
\operatorname{reg} S-t-1 \\
d-1
\end{array}\right) \cdot \ell(M) \\
& =e\left(\begin{array}{c}
\operatorname{reg} S-t-1 \\
d-1
\end{array}\right) \quad(\text { by Lemma } 3.2(\mathrm{i})) .
\end{aligned}
$$


Now we can bound the Hilbert cohomological functions of an arbitrary homogeneous ideal $I$. Recall that the defining degrees of $I$ are written in a decreasing sequence

and assume $\Delta \geq 2$.

$$
\Delta:=\delta_{1} \geq \delta_{2} \geq \cdots,
$$

In the proof of the following theorem, a result of Vasconcelos on the reduction number plays an essential role. We use initial ideals in order to go back to the situation of the previous result.

Theorem 3.4. Let $I$ be an arbitrary homogeneous ideal of $R$ and $S=R / I$. Let

$$
b=\min \left\{\delta_{1} \cdots \delta_{c},(\operatorname{adeg} I)^{c}\right\} .
$$

Then

(i) $h_{S}^{0}(t) \leq(b-1)\left(\begin{array}{c}t+d-2 \\ d-1\end{array}\right)$ for all $t \geq 0$.

(ii) For $1 \leq j \leq d-1$ and $t \leq \operatorname{reg} S$ :

$$
h_{S}^{j}(t) \leq(b-1)\left(\begin{array}{c}
\operatorname{reg} S+d-j-2 \\
d-j-1
\end{array}\right)\left(\begin{array}{c}
\operatorname{reg} S-t \\
j
\end{array}\right) .
$$

(iii) For $t<\operatorname{reg} S$ :

$$
h_{S}^{d}(t) \leq e\left(\begin{array}{c}
\operatorname{reg} S-t-1 \\
d-1
\end{array}\right) \leq b\left(\begin{array}{c}
\operatorname{reg} S-t-1 \\
d-1
\end{array}\right) .
$$

Proof. Let Gin $I$ denote the generic initial ideal of $I$ with respect to the reverse lexicographic order. Then Gin $I$ is a Borel-fixed ideal. Moreover we may assume that the coordinates $x_{1}, \ldots, x_{n}$ are chosen generically. By BS, Lemma 2.2 and Theorem 2.4] we have

$$
\ell\left(R /\left(I, x_{c+1}, \ldots, x_{n}\right)\right)=\ell\left(R /\left(\operatorname{Gin} I, x_{c+1}, \ldots, x_{n}\right)\right)
$$

and

$$
\operatorname{reg}(R / I)=\operatorname{reg}(R / \operatorname{Gin} I) .
$$

By Macaulay's theorem: $e(R / I)=e(R /$ Gin $I)$. Moreover, by [S, Theorem 2.4],

$$
h_{R / I}^{i}(t) \leq h_{R / \operatorname{Gin} I}^{i}(t)
$$

for all $i \geq 0$ and $t \in \mathbb{Z}$. Hence, the theorem immediately follows from the previous lemma if we can show that

$$
B:=\ell\left(R /\left(I, x_{c+1}, \ldots, x_{n}\right)\right) \leq b .
$$

a) Let $I^{\prime}$ denote the image of $I$ in $R^{\prime}=R /\left(x_{c+1}, \ldots, x_{n}\right) \cong K\left[x_{1}, \ldots, x_{c}\right]$. It is a $\left(x_{1}, \ldots, x_{c}\right)$-primary ideal. Since $I$ can be generated by elements of degrees $d_{1} \leq \delta_{1}, d_{2} \leq \delta_{2}, \ldots, I^{\prime}$ contains a regular sequence consisting of forms $f_{1}, \ldots, f_{c}$ of degrees $d_{1}^{\prime} \leq d_{1} \leq \delta_{1}, \ldots, d_{c}^{\prime} \leq d_{c} \leq \delta_{c}$. Hence

$$
B=\ell\left(R^{\prime} / I^{\prime}\right) \leq \ell\left(R^{\prime} /\left(f_{1}, \ldots, f_{c}\right)\right)=d_{1}^{\prime} \cdots d_{c}^{\prime} \leq \delta_{1} \cdots \delta_{c} .
$$

b) Since $x_{c+1}, \ldots, x_{n}$ is an s.o.p. of $R / \operatorname{Gin}(I)$, it is also an s.o.p. of $R / I$. Hence it is a minimal reduction of the algebra $R / I$. By $[\mathrm{V}$, Theorem 9.3.4],

$$
x_{i}^{\operatorname{adeg}(I)} \in\left(I, x_{c+1}, \ldots, x_{n}\right), \text { for all } i \geq 1 .
$$

This means $x_{1}^{\operatorname{adeg}(I)}, \ldots, x_{c}^{\operatorname{adeg}(I)}$ form a regular sequence in $I^{\prime}$. The above argument gives $B \leq(\operatorname{adeg} I)^{c}$. 
Remark 3.5. i) In the above theorem we may replace $b$ by $(\operatorname{reg} I)^{c}$ in order to get a bound for $h_{S}^{j}(t)$, which depends only on $\operatorname{reg} I$ and $d, c$.

ii) Hilbert cohomological functions are of reverse polynomial type; i.e., for each $i \geq 0$ there is a polynomial $p_{S}^{i}(t)$ such that $h_{S}^{i}(t)=p_{S}^{i}(t)$ for all $t \ll 0$ (see $\mathrm{BrS}$, Theorem 17.1.9]). The number

$$
\nu_{S}^{i}=\min \left\{t \in \mathbb{Z} ; h_{S}^{i}(t) \neq p_{S}^{i}(t)\right\}-1
$$

is called the $i$-th cohomological postulation number of $S$ (see $\mathrm{BrL}$ ). Thus, if $H_{\mathfrak{m}}^{i}(S), i<d$, is of finite length, then all graded components $H_{\mathfrak{m}}^{i}(S)_{t}$ vanish below $\nu_{S}^{i}$. Brodmann and Lashgari proved that all $-\nu_{S}^{i}, i \leq d$, can be bounded by a polynomial (of huge degree) in the numbers $h_{S}^{1}(0), \ldots, h_{S}^{d}(-d+1)$ (see BrL, Theorem 4.6]). Combining their result with Theorem 3.4 we see that $-\nu_{S}^{i}$ can be bounded by a polynomial in reg $S$. Thus, the number of "irregular" negative components of local cohomology modules is governed by the Castelnuovo-Mumford regularity.

\section{Hilbert coefficients}

Write the Hilbert polynomial in the form:

$$
P_{S}(t)=e_{0}\left(\begin{array}{c}
t+d-1 \\
d-1
\end{array}\right)-e_{1}\left(\begin{array}{c}
t+d-2 \\
d-2
\end{array}\right)+\cdots+(-1)^{d-1} e_{d-1} .
$$

Then $e_{0}, e_{1}, \ldots, e_{d-1}$ are called Hilbert coefficients of $S$. Note that $e_{0}=e$. Sometimes we also write $e_{i}=e_{i}(S)$ to emphasize its dependence on $S$.

We first estimate $\left|e_{i}\right|$ in terms of the arithmetic degree. For the application later, the following result is formulated in a rather technical way.

Theorem 4.1. Let $K$ be an infinite field and $I$ an arbitrary homogeneous ideal. Assume that $x_{c+1}, \ldots, x_{n}$ are chosen generically. Let $T_{d}=R /\left(I: \mathfrak{m}^{\infty}\right), T_{d-1}=$ $R /\left(\left(I, x_{n}\right): \mathfrak{m}^{\infty}\right), \ldots$ Then

(i) $\left|e_{1}\right| \leq(\operatorname{adeg} I)^{c}\left(\operatorname{reg} T_{2}+1\right) \leq(\operatorname{adeg} I)^{c} \operatorname{reg} I$.

(ii) For $i \geq 2,\left|e_{i}\right| \leq \frac{3}{2}(\operatorname{adeg} I)^{c}\left(\operatorname{reg} T_{i+1}+1\right)^{i} \leq \frac{3}{2}(\operatorname{adeg} I)^{c}(\operatorname{reg} I)^{i}$.

Proof. The second inequalities in both (i) and (ii) follow from Lemma 1.3(i). Let us prove the first ones. Set $T=T_{d}$. Let $H_{T}(t)$ denote the Hilbert function of $T$. Since $S$ and $T$ have the same Hilbert polynomial, $e_{i}=e_{i}(T)$ for all $i \geq 0$. From the Grothendieck-Serre formula

$$
P_{T}(t)-H_{T}(t)=\sum_{i=0}^{d}(-1)^{i+1} h_{T}^{i}(t),
$$

we get (setting $t=-1$ ),

$$
(-1)^{d-1} e_{d-1}=C-D,
$$

where

$$
C=h_{T}^{1}(-1)+h_{T}^{3}(-1) \cdots
$$

and

Hence

$$
D=h_{T}^{2}(-1)+h_{T}^{4}(-1) \cdots
$$

$$
\left|e_{d-1}\right| \leq \max \{C, D\} .
$$

For short, set $b=(\operatorname{adeg} I)^{c}$. If $d=2$, then by Theorem 3.4 we have $C=h_{T}^{1}(-1) \leq$ $(b-1)(\operatorname{reg} T+1)$ and $D=h_{T}^{2}(-1) \leq b \cdot \operatorname{reg} T$. Therefore $\left|e_{1}\right| \leq b \cdot\left(\operatorname{reg} T_{2}+1\right)$. 
Let $d \geq 3$. Since $x_{n}$ is generic, it is a regular element of $T$. Since $P_{T_{d-1}}(t)=$ $P_{T / x_{n} T}(t)$, we have $e_{i}=e_{i}(T)=e_{i}\left(T_{d-1}\right)$ for all $i \leq d-2$. The corresponding sequence of rings constructed for $T_{d-1}$ as above is exactly the rings $T_{d-1}, T_{d-2}, \ldots, T_{1}$. By Lemma 1.3(ii), $\operatorname{adeg} T_{d-1} \leq \operatorname{adeg} T$. Hence, by the induction hypothesis, it remains to prove (ii) for $i=d-1$. Note that

$$
\left(\begin{array}{c}
v+u-1 \\
u
\end{array}\right) \leq v^{u} \text { and }\left(\begin{array}{c}
v+1 \\
u
\end{array}\right) \leq(v+1) \frac{v^{u-1}}{u !} .
$$

Let $r=\operatorname{reg} T$. If $d=2 k+1$, where $k \geq 1$, then Theorem 3.4 yields

$$
\begin{aligned}
C & \leq(b-1)(r+1) r^{d-2}\left\{1+\frac{1}{3 !}+\cdots+\frac{1}{(2 k-1) !}\right\}+b \frac{r^{d-1}}{(2 k) !} \\
& \leq b(r+1) r^{d-2}\left\{1+\frac{1}{3 !}+\cdots+\frac{1}{(2 k-1) !}+\frac{1}{(2 k) !}\right\} \\
& \leq \frac{3}{2} b(r+1)^{d-1}
\end{aligned}
$$

and

$$
D \leq(b-1)(r+1) r^{d-2}\left\{\frac{1}{2 !}+\cdots+\frac{1}{(2 k) !}\right\} \leq(b-1)(r+1)^{d-1} .
$$

Hence $\left|e_{d-1}\right| \leq \frac{3}{2} b(r+1)^{d-1}$.

The inequality in the case $d=2 k, k \geq 2$, can be shown similarly.

Remark 4.2. a) In the above proof, if $h_{T}^{1}(-1)=0$, then $C \leq(\operatorname{adeg} I)^{c}(r+1)^{d-1}$. Hence, if $h_{T_{i+1}}^{1}(-1)=0$, then

$$
\left|e_{i}\right| \leq(\operatorname{adeg} I)^{c}\left(\operatorname{reg} T_{i+1}+1\right)^{i} \leq(\operatorname{adeg} I)^{c}(\operatorname{reg} I)^{i} .
$$

b) Consider again Example 1.9 $S=K[x, y, u, v] /\left((x, y)^{2}, x u^{t}+y v^{t}\right), t \geq 1$. We have $e_{1}=-(t+1)$, while $\operatorname{reg}(S)=t, \operatorname{adeg} S=2$ and the bound in (i) of the above theorem is $4(t+1)$. Thus one cannot avoid reg $I$ in the above theorem.

Note that $\operatorname{dim} T_{i+1}=i+1$. Combining Theorem 4.1 and Theorem 0.2 we get

Proposition 4.3. Let $S$ be a reduced ring of dimension at least two. Then

(i) $\left|e_{1}\right| \leq(\operatorname{adeg} S)^{c}\left(\frac{e(e-1)}{2}+\operatorname{adeg} S\right)$,

(ii) $\left|e_{i}\right| \leq \frac{3}{2}(\operatorname{adeg} S)^{c}\left(\frac{e(e-1)}{2}+\operatorname{adeg} S\right)^{i 2^{i-1}}$ if $i \geq 2$.

Another consequence of Theorem 4.1 is:

Corollary 4.4. Assume that $K$ is an algebraically closed field of characteristic 0 and $\operatorname{Proj}(R / I)$ is a reduced and pure-dimensional smooth subscheme in $\mathbb{P}^{n-1}$. Then for all $i \geq 1$ we have

$$
\left|e_{i}\right|<(i+2)^{i} e^{c+i}
$$

Proof. By Bertini's theorems (see [FOV] Corollary 3.4.6 and Corollary 3.4.14]) we may assume that all $\operatorname{Proj}\left(T_{i}\right)$ are reduced and pure-dimensional smooth subschemes. By Mumford's bound: $\operatorname{reg} T_{i+1} \leq(i+2)(e-2)+1$ (see [BM, Theorem 3.12(ii)]). Moreover, in this case $h_{T_{i+1}}^{1}(-1)=0$ for all $i \geq 1$ and adeg $I=e$. Hence, by Theorem 4.1 and Remark 4.2 , we get

$$
\left|e_{i}\right| \leq e^{c}((i+2)(e-2)+2)^{i}<(i+2)^{i} e^{c+i} .
$$


Remark 4.5. Let $S$ be a reduced ring of dimension at least two.

i) It is known that for any $K$-algebra $S, e_{1} \leq e(e-1) / 2$ (see [B1, Remark 3.10]). Hence in the statement (i) of Proposition 4.3 only the following inequality is new: $e_{1} \geq-(\operatorname{adeg} S)^{c}\left(\frac{e(e-1)}{2}+\operatorname{adeg} S\right)$.

ii) Let us recall

Eisenbud-Goto conjecture ([EG]). Let $K$ be an algebraically closed field. If $I$ is a prime-ideal containing no linear forms, then $\operatorname{reg} R / I \leq e-c$.

If this conjecture holds true, then by Remark 4.2 $\left|e_{i}\right| \leq(\operatorname{deg} S)^{c+i}$ provided $S$ is a domain. Note that the Eisenbud-Goto conjecture is close to being proved for smooth varieties of dimension at most 6 over a field of characteristic zero, by the work of several people including Lazarsfeld, Ran and Kwak. This indicates that the bounds in Theorem 0.2 and Proposition 4.3 are probably far from being sharp.

iii) There is a bound on $\left|e_{i}\right|$ in terms of the so-called homological degree, which also holds for any standard graded algebra over an Artinian ring; see RVV, Theorem 4.3]. However the homological degree is very large.

We now estimate $\left|e_{i}\right|$ by mean of the defining degrees. Recall that homogeneous elements $y_{1}, \ldots, y_{m}$ of $S$ form a filter regular sequence if $\left[\left(y_{1}, \ldots, y_{i-1}\right): y_{i}\right]_{t}=$ $\left(y_{1}, \ldots, y_{i-1}\right)_{t}$ for all $t \gg 0$ and $i=0, \ldots, m$. In other words, $y_{i}$ is a filter regular element on $S /\left(y_{1}, \ldots, y_{i-1}\right) S$.

Theorem 4.6. Let $I$ be an arbitrary homogeneous ideal. Assume that $d \geq 2$ and $x_{c+1}, \ldots, x_{n}$ is a filter regular sequence on $S$. Let $S_{d}=S, S_{d-1}=S / x_{n} S_{d}, \ldots$. Set $\pi=\delta_{1} \cdots \delta_{c}$. Then

(i) $\left|e_{1}\right| \leq \pi \cdot\left(\operatorname{reg} S_{2}+1\right) \leq \pi \cdot \operatorname{reg} I$.

(ii) For $i \geq 2,\left|e_{i}\right| \leq \frac{3}{2} \pi \cdot\left(\operatorname{reg} S_{i+1}+1\right)^{i} \leq \frac{3}{2} \pi \cdot(\operatorname{reg} I)^{i}$.

Proof. The proof is similar to that of Theorem 4.1 after noticing that $\operatorname{reg} S_{i} \leq$ $\operatorname{reg} S_{i+1}$ and $\delta_{1}\left(S_{i}\right) \leq \delta_{1}\left(S_{i+1}\right), \ldots, \delta_{c}\left(S_{i}\right) \leq \delta_{c}\left(S_{i+1}\right)$ for all $i \geq 2$.

Combining it with Theorem 2.1 we immediately get

Proposition 4.7. Let $d \geq 1$. Then

(i) $\left|e_{1}\right| \leq \pi(\pi+\Delta-1)^{2}$.

(ii) For all $i \geq 2$, we have $\left|e_{i}\right| \leq \frac{3}{2} \pi(\pi+\Delta-1)^{i 2^{i}}$.

In particular $\left|e_{i}\right|<\left(\frac{3}{2} \Delta^{c}+\Delta\right)^{1+i 2^{i}}$ for all $i \geq 1$.

A direct application of Theorem 4.6 sometimes gives much better bounds than the ones in the previous proposition. For example, using BEL and the second inequality in Theorem 4.6(ii), one immediately gets that

$$
\left|e_{i}\right| \leq \frac{3}{2} \pi(\operatorname{reg} I)^{i} \leq \frac{3}{2} \Delta^{c}(c(\Delta-1))^{i}<\frac{3}{2} c^{i} \Delta^{c+i},
$$

provided $\operatorname{Proj}(R / I)$ is a reduced and pure-dimensional smooth subscheme. Another case is

Corollary 4.8. Let $I$ be an ideal generated by monomials of degree at most $\Delta$ in $n$ variables. Then for all $i \geq 1$ we have

$$
\left|e_{i}\right| \leq \frac{3}{2} \min \left\{(\operatorname{adeg} I)^{c+i}, n^{i} \Delta^{c+i}\right\} .
$$


Proof. By [HT, Theorem 1.1], reg $I \leq \operatorname{adeg} I$ and by Taylor's resolution (see also [HT, Theorem 1.2]), reg $I \leq n \Delta$. Hence the statement follows from Theorems 4.1 and 4.6 .

The following example shows that the bounds in Theorem 4.6 and Corollary 4.8 are rather good.

Example 4.9. Let $n>c+1$ and

$$
I=\left(x_{1}, \ldots, x_{c}\right) \cap\left(x_{1}^{r}, \ldots, x_{c}^{r}, x_{c+1}^{r-1}, \ldots, x_{n-1}^{r-1}\right) .
$$

Using the exact sequence

$$
0 \rightarrow R / I \rightarrow R / P \oplus R / J \rightarrow R /(P+J) \rightarrow 0,
$$

where $P=\left(x_{1}, \ldots, x_{c}\right)$ and $J=\left(x_{1}^{r}, \ldots, x_{c}^{r}, x_{c+1}^{r-1}, \ldots, x_{n-1}^{r-1}\right)$, one can check that

$$
\operatorname{reg} R / I=(n-1) r-2 n+c+2
$$

and

$$
P_{R / I}(t)=\left(\begin{array}{c}
t+d-1 \\
d-1
\end{array}\right)+\left[r^{c}(r-1)^{d-1}-(r-1)^{d-1}\right] .
$$

Hence $\left|e_{d-1}\right|=\left(r^{c}-1\right)(r-1)^{d-1}$, while by Corollary $4.8,\left|e_{d-1}\right| \leq 3 r^{c+d-1} n^{d-1} / 2$, and by Theorem 4.6] $\left|e_{d-1}\right| \leq 3 r^{c}[(n-1) r-2 n+c+3]^{d-1} / 2$.

\section{Finiteness of Hilbert functions}

In this section we prove Theorem 0.1 . We need some further preliminary results. The following result extends an estimation of $H_{S}(t)$ mentioned in Remark [1.6 to arbitrary ideals.

Lemma 5.1. Let I be an arbitrary homogeneous ideal. Let

$$
b=\min \left\{\delta_{1} \cdots \delta_{c},(\operatorname{adeg} S)^{c}\right\} .
$$

For all $t \geq 0$ we have

$$
H_{S}(t) \leq(b-1)\left(\begin{array}{c}
t+d-2 \\
d-1
\end{array}\right)+\left(\begin{array}{c}
t+d-1 \\
d-1
\end{array}\right) .
$$

Proof. We may assume that $x_{c+1}, \ldots, x_{n}$ are chosen generically. In particular, $x_{c+1}, \ldots, x_{n}$ form an s.o.p. of $S$. Set $B=\ell\left(S /\left(x_{c+1}, \ldots, x_{n}\right) S\right)$. By [RVV] Proposition 2.4] for all $t \geq 0$ we have

$$
H_{S}(t) \leq(B-1)\left(\begin{array}{c}
t+d-2 \\
d-1
\end{array}\right)+\left(\begin{array}{c}
t+d-1 \\
d-1
\end{array}\right)
$$

As shown in the proof of Theorem 3.4, $B \leq b$. Hence the lemma is proven.

Lemma 5.2. Assume that $K$ is an algebraically closed field, $I$ is an intersection of prime ideals and $I$ contains no linear form. Then $c \leq d(\operatorname{adeg} I-1)$.

Proof. By the assumption,

$$
I=\bigcap_{i=1}^{s} \mathfrak{p}_{i},
$$

where $\mathfrak{p}_{i}$ are prime ideals of height at least $c$. Since $s \leq \operatorname{adeg} I$, the statement is derived from the following inequality:

$$
c \leq \operatorname{adeg} I-s+(s-1) d .
$$


We prove this inequality by induction on $s$. The case $s=1$ is well known. Let $s>1$. Put $J=\bigcap_{i=1}^{s-1} \mathfrak{p}_{i}$. Let $a$ and $b$ be the maximal number of independent linear forms contained in $J$ and $\mathfrak{p}_{s}$, respectively. By the induction hypothesis, we have $c-a \leq \operatorname{adeg} J-(s-1)+(s-2) d$ and $c-b \leq e\left(R / \mathfrak{p}_{s}\right)-1$. Since $\operatorname{adeg} I=\operatorname{adeg} J+e\left(R / \mathfrak{p}_{s}\right)$, we get

$$
2 c \leq \operatorname{adeg} I-s+(s-2) d+a+b .
$$

If $a+b>n$, it would imply that there is a linear form in $J \cap \mathfrak{p}_{s}=I$, a contradiction. Hence $a+b \leq n=d+c$. The above inequality then yields $c \leq \operatorname{adeg} I-s+(s-1) d$.

As mentioned in the introduction, Theorem 0.1 is an immediate consequence of Theorem 0.2. Lemma 5.2 and [RTV2, Theorem 2.3]. We give here a direct proof without the use of [RTV2].

Proof of Theorem 0.1. Without loss of generality, we may assume from the beginning that $I$ contains no linear form. Note that $e \leq \operatorname{adeg} S$. Therefore, by Proposition 4.3 and Lemma 5.2, there are only finitely many Hilbert polynomials associated with reduced algebras such that $\operatorname{adeg} S \leq a$ and $\operatorname{dim} S \leq d$. By Lemmas 5.1 and 5.2. there are only finitely many choices for the initial values of Hilbert functions, while Theorem 0.2 says that for $t \geq\left(\frac{e(e-1)}{2}+\operatorname{adeg} S\right)^{2^{d-2}}$ each Hilbert function agrees with the corresponding Hilbert polynomial. This implies the finiteness of the number of Hilbert functions.

Example 1.9] shows that without the assumption of $S$ being a reduced ring, Theorem 0.1 does not hold.

Applying Proposition 4.7 and Theorem 2.1, as in the proof of Theorem 0.1 , we get a similar finiteness result in terms of the defining degrees.

Corollary 5.3. Given two numbers $\delta$ and $n$, there exist only finitely many Hilbert functions associated with homogeneous ideals generated by forms of degrees at most $\delta$ in at most $n$ variables.

\section{Castelnuovo-Mumford Regularity of initial ideals}

In this last section we apply the results in the previous sections to study the Castelnuovo-Mumford regularity of an initial ideal in $(I)$ of $I$ with respect to any given term order and coordinates. We even consider a much larger class: the class of all ideals $J$ having the same Hilbert function as $I$. Then one can easily bound reg $J$ in terms of some data of $I$. This approach was initiated in [CM] and developed further in $[\mathrm{HH}]$. Let us recall some notation. The Hilbert polynomial can be uniquely written in the form

$$
P_{R / I}(t)=\left(\begin{array}{c}
c_{1}+t \\
t
\end{array}\right)+\left(\begin{array}{c}
c_{2}+t-1 \\
t-1
\end{array}\right)+\cdots+\left(\begin{array}{c}
c_{s}+t-s+1 \\
t-s+1
\end{array}\right),
$$

where $c_{1} \geq c_{2} \geq \cdots \geq c_{s} \geq 0$ are integers (see, e.g., $[\mathrm{V}$, Section B6]). For $0 \leq i \leq d-1$ set

$$
B_{i}=\sharp\left\{j ; c_{j} \geq(d-1)-i\right\} .
$$


Thus in the above notation, $s=B_{d-1}$ (for convenience, we set $B_{-1}=0$ ). The following result easily follows from Gotzmann's regularity theorem:

Lemma 6.1 ([ $[\mathrm{HH}$, Lemma 5]). Let $I, J$ be homogeneous ideals having the same Hilbert function. Then

$$
\operatorname{reg} J \leq \max \left\{\operatorname{reg} I, B_{d-1}\right\} .
$$

Since we already know bounds for $\operatorname{reg}(I)$ (see Theorems 0.2 and 2.1), we have only to estimate $B_{d-1}$. For this purpose we need some relations between the invariants $B_{i}$ just defined and the Hilbert coefficients which were given in [B], Proposition 3.9] (see also [CM, Lemma 1.5]).

Lemma 6.2. For all $0 \leq j \leq d-1$ we have

$$
B_{i}=(-1)^{i} e_{i}+\left(\begin{array}{c}
B_{i-1}+1 \\
2
\end{array}\right)-\left(\begin{array}{c}
B_{i-2}+1 \\
3
\end{array}\right)+\cdots+(-1)^{i+1}\left(\begin{array}{c}
B_{0}+1 \\
i+1
\end{array}\right) .
$$

Note that $B_{d-1} \geq \cdots \geq B_{0}=e$. In order to estimate $B_{j}$, we need the following combinatorial result.

Lemma 6.3. Assume that

$$
\left|e_{i}\right| \leq M^{\alpha+i \beta 2^{i}} \quad \text { for all } i \geq 0,
$$

where $M \geq 2$ and $\alpha, \beta \geq 1$. Then for all $0 \leq j \leq d-1$ we have

$$
B_{j} \leq M^{(\alpha+j \beta) 2^{j}}
$$

Proof. We have $B_{0}=e=e_{0} \leq M^{\alpha}$ by the assumption. By Lemma6.2 the following holds:

$B_{1}=-e_{1}+\left(\begin{array}{c}B_{0}+1 \\ 2\end{array}\right) \leq\left|e_{1}\right|+\frac{e(e+1)}{2}<M^{\alpha+2 \beta}+M^{2 \alpha} \leq M^{2(\alpha+\beta)} \quad($ since $M \geq 2)$.

Let $j \geq 2$. Assume that

$$
B_{j-l} \leq M^{(\alpha+(j-l) \beta) 2^{j-l}}
$$

for all $l \geq 1$. Lemma 6.2 yields:

$$
\begin{aligned}
B_{j} & =(-1)^{j} e_{j}+\left(\begin{array}{c}
B_{j-1}+1 \\
2
\end{array}\right)-\left(\begin{array}{c}
B_{j-2}+1 \\
3
\end{array}\right)+\cdots+(-1)^{j-1}\left(\begin{array}{c}
B_{0}+1 \\
j-1
\end{array}\right) \\
& \leq\left|e_{j}\right|+\left(\begin{array}{c}
B_{j-1}+1 \\
2
\end{array}\right)+\left(\begin{array}{c}
B_{j-3}+1 \\
4
\end{array}\right)+\cdots .
\end{aligned}
$$

By (10), for all $1 \leq l \leq j$ we have

$$
\left(\begin{array}{c}
B_{j-l}+1 \\
l+1
\end{array}\right) \leq \frac{\left(B_{j-l}+1\right)^{l+1}}{(l+1) !} \leq \frac{\left(B_{j-l}+1\right)^{2^{l}}}{(l+1) !} \leq \frac{M^{(\alpha+j \beta) 2^{j}}}{(l+1) !} .
$$

From (11), (12) and the assumption $\left|e_{j}\right| \leq M^{\alpha+j \beta 2^{j}}$ it follows that

$$
\begin{aligned}
B_{j} & \leq M^{\alpha+j \beta 2^{j}}+M^{(\alpha+j \beta) 2^{j}}\left\{\frac{1}{2 !}+\frac{1}{4 !}+\cdots\right\} \\
& <M^{\alpha+j \beta 2^{j}}+\frac{2}{3} M^{(\alpha+j \beta) 2^{j}} \\
& \leq M^{(\alpha+j \beta) 2^{j}} .
\end{aligned}
$$


By Macaulay's theorem $H_{R / \text { in } I}(t)=H_{R / I}(t)$ for all $t \in \mathbb{Z}$. Hence, Theorem 0.3 stated in the introduction is a special case of the following result.

Theorem 6.4. Let $K$ be an arbitrary field. Let $J$ be an arbitrary homogeneous ideal of $R=K\left[x_{1}, \ldots, x_{n}\right]$ such that $H_{R / J}(t)=H_{R / I}(t)$ for all $t$. Then

(i) $\operatorname{reg}(J) \leq\left(\frac{3}{2} \Delta^{c}+\Delta\right) d 2^{d-1}$.

(ii) Moreover, if $R / I$ is a reduced algebra, then we also have

$$
\operatorname{reg}(J) \leq(\operatorname{adeg}(I))^{(n-1) 2^{d-1}} .
$$

Proof. (i) By Proposition 4.7, $\left|e_{i}\right|<\left(\frac{3}{2} \Delta^{c}+\Delta\right)^{1+i 2^{i}}$ for all $i \geq 0$. Applying Lemma 6.3 to $M=\frac{3}{2} \Delta^{c}+\Delta, \alpha=1, \beta=1$ and $j=d-1$, we get $B_{d-1} \leq\left(\frac{3}{2} \Delta^{c}+\Delta\right)^{d 2^{d-1}}$. Then (i) follows from Lemma 6.1 and Theorem 2.1

(ii) For short, set $a=\operatorname{adeg} I$. Note that $a \geq e$ and

$$
\frac{e(e-1)}{2}+a \leq a^{2} .
$$

Hence, by Proposition 4.3 (i),

$$
\left|e_{1}\right| \leq a^{c+2} .
$$

Let $i \geq 2$. Since $\Delta \geq 2, a \geq 2$. By Proposition 4.3)(ii) and (13), we have

$$
\begin{aligned}
\left|e_{i}\right| & \leq \frac{3}{2} a^{c}\left(\frac{a(a+1)}{2}\right)^{i 2^{i-1}}=a^{c}\left(\frac{a(a+1)}{2}\right)^{i 2^{i-1}-4} \cdot\left[\frac{3}{2}\left(\frac{a(a+1)}{2}\right)^{4}\right] \\
& \leq a^{c+2\left(i 2^{i-1}-4\right)} a^{8}=a^{c+i 2^{i}} .
\end{aligned}
$$

Thus, applying Lemma 6.3 to $M=a, \alpha=c, \beta=1$ and $j=d-1$, we get $B_{d-1} \leq$ $a^{(n-1) 2^{d-1}}$. By Lemma 6.1 and Theorem 0.2 this implies reg $J \leq a^{(n-1) 2^{d-1}}$.

Note that if $R / I$ is a Cohen-Macaulay ring of dimension $d \geq 2$ (but not necessarily reduced), then one can get a little bit better bound (see $\mathrm{HH}$, Theorem 9]):

$$
\operatorname{reg} J \leq e^{2^{d-1}} / 2^{2^{d-2}} .
$$

Example 6.5. Let $I^{\text {lex }}$ denote the lex-segment ideal associated to the Hilbert function $H_{R / I}(t)$. This is the ideal generated by all first $H_{I}(m)$ monomials of degrees $m$ with respect to the lexicographic order, when $m$ runs through all positive integers. It has the same Hilbert function as $I$. If $R / I$ is a Cohen-Macaulay ring of dimension $d \geq 2$, then from [CM, Theorem 2.5] it follows that $\operatorname{reg}\left(I^{\text {lex }}\right)=B_{d-1}$.

i) Let $I$ be an ideal generated by a regular sequence consisting of forms of degrees $\delta_{1} \geq \cdots \geq \delta_{c}$ such that $c \geq 2$ and $\delta_{2} \geq 35(d \geq 2)$. It was shown in $\underline{\mathrm{HH}}$, Example 13] that

$$
\operatorname{reg}\left(I^{l e x}\right) \geq 9 \frac{\Delta^{c 2^{d-1}}}{9^{2^{d-2}}} .
$$

This shows that the bound in Theorem 6.4(i) is close to being sharp.

ii) Let $S$ be a Veronesian embedding $K\left[y_{1}, \ldots, y_{d}\right]^{(p)}$; i.e., $S_{1}$ is generated by all monomials of degree $p$ in the variables $y_{1}, \ldots, y_{d}$, where $d \geq 3$. This is a CohenMacaulay domain and $P_{S}(t)=\left(\begin{array}{c}p t+d-1 \\ d-1\end{array}\right)$. Hence adeg $S=e=p^{d-1}$ and $e_{1}=$ $d p^{d-2}(p-1)$. Let $p \geq 35$. Then $e_{1}<e^{2} / 36$ and $e \geq 35^{2}$. Let $S=K\left[x_{1}, \ldots, x_{q}\right] / I$, where $q=\left(\begin{array}{c}p+d-1 \\ d-1\end{array}\right)$. By [ $[\mathrm{HH}$, Proposition 12] we get

$$
\operatorname{reg}\left(I^{l e x}\right) \geq 9 \frac{e^{2^{d-1}}}{9^{2^{d-2}}} .
$$


This shows that the bound in the second part of Theorem 6.4 is close to being sharp too.

Since reg(in $I) \geq \operatorname{reg} I$, the ideals of Mayr and Meyer again show that the bound $\left(2 \Delta^{c}\right)^{d 2^{d-1}}$ of Theorem 0.3 is rather good (see Remark 2.6). We do not know whether one can construct a reduced algebra $R / I$ such that there is a term order with reg(in $I)$ close to $(\operatorname{adeg}(I))^{(n-1) 2^{d-1}}$.

Finally we would like to make the following remark: In the proof of Theorem 0.3 we use a very rough estimation for $\operatorname{reg} I$ and $\left|e_{i}\right|$. One could suspect that if reg $I$ and $\left|e_{i}\right|$ are small, then one could get a bound for reg(in $I$ ), which would be a single exponent of $d$. But this is not the case as shown by [HH, Section 4].

\section{ACKNOWLEDGMENT}

The author would like to thank the Centre de Recerca Matematica (Spain) for the financial support and hospitality during the final preparation of this article. He is grateful to the referee for his/her useful remarks and suggestions, which led to an improvement of some main results of the paper.

\section{ADDED IN PROOF}

After submitting this paper for publication, the author found that the main result of the following paper is a weaker version of the first statement of Theorem 0.3. T. W. Dubé, The structure of polynomial ideals and Gröbner bases, SIAM J. Comput. 19 (1990), no. 4, 750-773. The approach there is purely combinatorial.

\section{REFERENCES}

[BM] D. Bayer and D. Mumford, What can be computed in algebraic geometry?, Computational algebraic geometry and commutative algebra (Cortona, 1991), 1-48, Sympos. Math., XXXIV, Cambridge Univ. Press, Cambridge, 1993. MR95d:13032

[BS] D. Bayer and M. Stillman, A criterion for detecting m-regularity, Invent. Math. 87(1987), no. 1, 1-11. MR:87k:13019

[BEL] A. Bertram, L. Ein and R. Lazarsfeld, Vanishing theorems, a theorem of Severi, and the equations defining projective varieties, J. Amer. Math. Soc. 4(1991), 587-602. MR 92g:14014

[Bl] C. Blancafort, Hilbert functions of graded algebras over Artinian rings, J. Pure Appl. Algebra 125(1998), no. 1-3, 55-78. MR98m:13023

[BrL] M. P. Brodmann and A. F. Lashgari, A diagonal bound for cohomological postulation numbers of projective schemes, J. Algebra 265(2003), 631-650. MR2004f:14030

[BrS] M. P. Brodmann and R. Y. Sharp, Local cohomology: an algebraic introduction with geometric applications. Cambridge Studies in Advanced Mathematics, 60. Cambridge University Press, Cambridge, 1998. MR99h:13020

[CS] G. Caviglia and E. Sbarra, Characteristic-free bounds for Castelnuovo-Mumford regularity, Compos. Math. 141 (2005), no. 6, 1365-1373. MR2006i:13032

[CF] M. Chardin and A. L. Fall, Sur la régularité de Castelnuovo-Mumford de idéaux en dimension 2, C. R. Math. Acad. Sci. Paris 341 (2005), no. 4, 233-238. MR2164678

[CM] M. Chardin and G. Moreno-Socias, Regularity of lex-segment ideals: Some closed formulas and applications, Proc. Amer. Math. Soc. 131(2003), no. 4, 1093-1102. MR:2003m:13014

[E] D. Eisenbud, Commutative algebra. With a view toward algebraic geometry. Graduate Texts in Mathematics, 150. Springer-Verlag, New York, 1995. MR97a:13001

[EG] D. Eisenbud and S. Goto, Linear free resolutions and minimal multiplicity, J. Algebra 88(1984), no. 1, 89-133. MR 85f:13023

[FOV] H. Flenner, L. O'Carroll and W. Vogel, Joins and intersections. Springer Monographs in Mathematics. Springer-Verlag, Berlin, 1999. MR2001b:14010 
[Gi] M. Giusti, Some effectivity problems in polynomial ideal theory. EUROSAM 84 (Cambridge, 1984), 159-171, Lecture Notes in Comput. Sci., 174, Springer, Berlin, 1984. MR779123 (86d:12001)

[GLP] L. Gruson, R. Lazarsfeld and C. Peskine, On a theorem of Castelnuovo, and the equations defining space curves, Invent. Math. 72(1983), no. 3, 491-506. MR85g:14033

[HPV] J. Herzog, D. Popescu and M. Vladoiu, On the Ext-modules of ideals of Borel type, Commutative algebra (Grenoble/Lyon, 2001), 171-186, Contemp. Math., 331, Amer. Math. Soc., Providence, RI, 2003. MR2013165

[HH] L. T. Hoa and E. Hyry, Castelnuovo-Mumford regularity of initial ideals, J. Symb. Comp. 38(2004), 1327-1341. MR2168718

[HSV] L. T. Hoa, J. Stückrad and W. Vogel, Towards a structure theory for projective varieties of degree $=$ codimension +2 , J. Pure Appl. Algebra, 71, 203-231(1991). MR1117635 (92f:14002)

[HT] L. T. Hoa; N. V. Trung, On the Castelnuovo-Mumford regularity and the arithmetic degree of monomial ideals, Math. Z. 229(1998), no. 3, 519-537. MR99k:13034

[K] S. L. Kleiman, Les théorèmes de finitude pour le foncteur de Picard, in: "Théorie des intersections et théorème de Riemann-Roch" (French) Séminaire de Géométrie Algébrique du Bois-Marie 1966-1967 (SGA 6), pp. 616-666. Lecture Notes in Mathematics, Vol. 225. Springer-Verlag, Berlin-New York, 1971. MR50:7133

[MVY] C. Miyazaki, W. Vogel and K. Yanagawa, Associated primes and arithmetic degrees, J. Algebra 192(1997), no. 1, 166-182. MR98i:13036

[MM] H. M. Möller and F. Mora, Upper and lower bounds for the degree of Gröbner bases, EUROSAM 84 (Cambridge, 1984), 172-183, Lecture Notes in Comput. Sci., 174, Springer, Berlin, 1984. MR86k:13008

[M] D. Mumford, Lectures on curves on an algebraic surface, Princeton Univ. Press, Princeton, 1966. MR 35:187

[RTV1] M. E. Rossi, N. V. Trung and G. Valla, Castelnuovo-Mumford regularity and extended degree, Trans. Amer. Math. Soc. 355(2003), no. 5, 1773-1786. MR2004b:13020

[RTV2] M. E. Rossi, N. V. Trung and G. Valla, Castelnuovo-Mumford regularity and finiteness of Hilbert functions, Lect. Notes Pure Appl. Math., 244, Chapman \& Hall/CRC, Boca Raton, FL, 2006. MR2184798 (2006k:13040)

[RVV] M. E. Rossi, G. Valla and W. V. Vasconcelos, Maximal Hilbert functions, Results Math. 39(2001), 99-114. MR:1817403(2001m:13020)

[S] E. Sbarra, Upper bounds for local cohomology for rings with given Hilbert function, Comm. Algebra 29(2001), no. 12, 5383-5409. MR2002j:13024

[Sj] R. Sjögren, On the regularity of graded $k$-algebras of Krull dimension $\leq 1$, Math. Scand. 71(1992), 167-172. MR94b:13010

[V] W. V. Vasconcelos, Computational methods in commutative algebra and algebraic geometry. With chapters by D. Eisenbud, D. R. Grayson, J. Herzog and M. Stillman. Algorithms and Computation in Mathematics, 2. Springer-Verlag, Berlin, 1998. MR.99c:13048

Institute of Mathematics, 18 Hoang Quoc Viet Road, 10307 Hanoi, Vietnam

E-mail address: 1thoa@math.ac.vn 